\title{
Desempenho de frangos caipiras alimentados com rações contendo diferentes níveis de energia metabolizável
}

\author{
[Performance of broiler chickens fed diets containing different levels \\ of metabolizable energy] \\ A.S. Moreira ${ }^{1}$, M.S.V. Santos ${ }^{2}$, S. S. Vieira ${ }^{1}$, F.B. Tavares $^{1}$, M.C. Manno ${ }^{3}$ \\ ${ }^{1}$ Alunos de pós-graduação - PPGSPAA - UFRA - Belém, PA \\ ${ }^{2}$ Universidade Federal Rural da Amazônia - UFRA - Parauapebas, PA \\ ${ }^{3}$ Universidade Federal Rural da Amazônia, UFRA - Belém, PA
}

\begin{abstract}
RESUMO
Analisaram-se os efeitos da energia metabolizável (EM) e da idade de abate sobre o desempenho zootécnico, o rendimento de carcaça e de partes de frangos da linhagem Caipira Francês Exótico. Foram utilizadas 192 aves, distribuídas em delineamento inteiramente ao acaso, com três tratamentos e quatro repetições de 16 aves cada. Os tratamentos (T) foram definidos de acordo com os níveis de energia das dietas inicial ( 1 a 28 dias) e final (29 a 90 dias), respectivamente, em: T1 - 3.000 e 3.100kcal de EM/kg; $\mathrm{T} 2-3.100$ e $3.200 \mathrm{kcal}$ de EM/kg; T3 - 3.200 e $3.300 \mathrm{kcal}$ de EM $/ \mathrm{kg}$. Os abates foram realizados aos 77, 84 e 90 dias. Na fase inicial, as aves do T2 e do T3 apresentaram maior peso médio $(655,75$ e 713,50g), maior ganho de peso (24,28 e 26,42g/ave) e melhor conversão alimentar (1,59 e 1,77), e não diferiram entre si $(\mathrm{P}>0,05)$. Quando abatidas aos 77 dias de idade, as aves que consumiram a dieta com $3.100 / 3.200 \mathrm{kcal}$ de $\mathrm{EM} / \mathrm{kg}$ apresentaram maior rendimento de carcaça $(82,20 \%)$ e de peito $(24,26 \%)$, e não diferiram dos frangos alimentados com dietas contendo 3.000/3.200kcal de EM/kg, nos quais se verificaram rendimento de carcaça de $80,72 \%$ e $23,20 \%$ de rendimento de peito. Os frangos da linhagem Caipira Francês Exótico apresentaram melhores desempenho zootécnico e rendimento de carcaça quando alimentados com dietas contendo 3.100 a $3.200 \mathrm{kcal}$ de EM/kg na ração e abatidos aos 77 dias de idade.
\end{abstract}

Palavras-chave: desempenho, exigência nutricional, master gris, rendimento de carcaça

\begin{abstract}
This is study aimed to analyze the effects of metabolizable energy (ME) and age at slaughter on growth performance, carcass yield and prime cuts, relative weight of the wings, back, head + neck and feet of broiler chickens from the Caipira Francês Exótico strain. A total of 192 birds were distributed in a completely randomized design with three treatments and four replicates of 16 birds per treatment. The treatments were defined according to levels of dietary energy ( 1 to 28 days) and late (29-90 days), respectively: T1 - 3000 and $3100 \mathrm{kcal} / \mathrm{kg} \mathrm{ME}, \mathrm{T2}-3100$ and $3200 \mathrm{kcal} / \mathrm{kg} \mathrm{ME}, \mathrm{T3}-3200$ and $3300 \mathrm{kcal} / \mathrm{kg}$ ME. The slaughter was carried out at 77, 84 and 90 days. In the initial phase the birds from T2 and T3 had a higher mean weight (655.75 and 713.50g), weight gain (24.28 and $26.42 \mathrm{~g} / \mathrm{bird})$ and better feed gain (1.59 and 1.77) and did not differ ( $P>0.05)$. When slaughtered at 77 days of age, birds fed the diet with 3.100/3.200kcal/kg ME had higher carcass yield (82.20\%) and breast (24.26\%), and did not differ from chickens fed diets containing 3.000/3.200kcal/ $\mathrm{kg} M E$, where a carcass yield of $80.72 \%$ and $23.20 \%$ of breast yield was found. The Caipira Francês Exótico broilers had better growth performance and carcass yield than when fed diets containing 3100-3200kcal/kg in the diet and slaughtered at 77 days old.
\end{abstract}

Keywords: broilers, carcass yield, dietary energy, master gris, performance

Recebido em 18 de março de 2011

Aceito em 15 de maio de 2012

E-mail: allynemoreira@zootecnista.com.br

Apoio: Fundação de Amparo à Pesquisa do Estado do Pará - FAPESPA 


\section{INTRODUÇÃO}

A avicultura é uma das atividades de produção animal que mais se desenvolveu nos últimos anos. Isto se deve, basicamente, ao melhoramento genético das diferentes linhagens de aves domésticas, da formulação e elaboração de dietas e da busca de novos sistemas de criação que objetivam maior produtividade no menor tempo possível (Viana et al., 2000).

Entre os mercados de produtos avícolas, existe um segmento diferenciado, composto por consumidores mais exigentes, que demandam por produtos mais saborosos, mais firmes e com sabor diferenciado (Bastianelli, 2001). Trata-se da produção de frangos caipiras, uma atividade produtiva que representa uma oportunidade para complementar as atividades pecuárias desenvolvidas nas propriedades rurais e urbanas (Figueiredo et al., 2001). Essa capacidade de integração de criação de frangos com outras atividades agrícolas, agroindustriais, extrativistas e pecuárias que são costumeiramente desenvolvidas pelo agricultor familiar, resulta na agregação de valor e em maior remuneração por produto acabado (Sagrilo, 2002).

$\mathrm{Na}$ avicultura alternativa, utilizam-se aves com características próprias, sendo que estas, normalmente, apresentam curvas e taxa de crescimento diferentes das linhagens comerciais de corte. Geralmente, são aves de crescimento mais lento, cujas exigências nutricionais devem diferir das exigências dos frangos de corte de linhagens industriais (Mendonça et al., 2007). Embora a produção de aves criadas em sistemas alternativos tenha aumentado consideravelmente nos últimos anos, ainda são incipientes as realizações de trabalhos de pesquisa que avaliem os parâmetros nutricionais e seus efeitos sobre o desempenho nas aves híbridas tipo caipira no Brasil.

Com a introdução dessas aves de linhagens de crescimento lento no mercado brasileiro, o setor adotou novos critérios de manejo e nutrição de frangos de corte, a fim de maximizar a produtividade e otimizar os custos. Com isso, a principal preocupação durante a formulação de rações para frangos de corte é fornecer energia em quantidade adequada, visto que o desempenho zootécnico das aves está correlacionado com os níveis energéticos das dietas (Freitas et al., 2006). Segundo Lesson e Summers (2001), esse nível energético afeta tanto o desempenho biológico quanto o econômico. Quando se aumenta o nível energético da dieta sem o adequado ajuste de nutrientes, ocorre desequilíbrio de alguns deles, o que provoca deposição excessiva de gordura na carcaça e diminuição da taxa de crescimento.

O objetivo deste estudo foi avaliar os efeitos dos níveis de energia metabolizável da dieta e da idade de abate sobre o desempenho zootécnico, o rendimento de carcaça e de cortes nobres, o peso relativo das asas, do dorso, da cabeça+pescoço e pés de frangos de corte da linhagem Caipira Francês Exótico (Master Gris Cou Plumé).

\section{MATERIAL E MÉTODOS}

Foram alojados 192 pintos de corte da linhagem Caipira Francês Exótico (Master Gris Cou Plumé), mistos, com um dia de idade. As aves foram distribuídas em boxes, contendo 16 animais cada, criados em sistema intensivo, com densidade de 6,4 aves $/ \mathrm{m}^{2}$. O período experimental teve duração de 90 dias.

O delineamento experimental foi inteiramente ao acaso, com três tratamentos e quatro repetições, sendo a unidade experimental um box com 16 aves. Os tratamentos foram definidos de acordo com os níveis de energia metabolizável das rações inicial (1 a 28 dias) e final (29 a 90 dias), respectivamente: $\mathrm{T} 1-3.000$ e $3.100 \mathrm{kcal}$ de $\mathrm{EM} / \mathrm{kg}$; T2 - 3.100 e $3.200 \mathrm{kcal}$ de EM/kg e T3 3.200 e $3.300 \mathrm{kcal}$ de EM/kg.

As dietas foram formuladas à base de milho moído, farelo de soja, farinha de carne e ossos, óleo de soja, calcário e sal. O atendimento das necessidades em vitaminas e minerais foi possível por meio da adição de premix comercial, recomendado para frango de corte nas fases inicial e final. Na elaboração das dietas experimentais, foi utilizado um programa de formulação de rações, sendo formuladas de modo a serem isoproteicas, isoaminoacídicas e isofosfóricas. A composição das dietas experimentais nas fases inicial e de engorda está apresentada nas Tab. 1 e 2, respectivamente. 
Antes do início do experimento, o galpão e os equipamentos foram limpos e desinfetados. Os pintos foram vacinados no incubatório contra a doença de Marek e a Bouba Aviária e, aos sete dias de idade, receberam a vacina contra Newcastle. No alojamento, 16 pintos foram pesados de forma homogênea para a composição da parcela experimental, distribuídos nos boxes de acordo com a identificação dos tratamentos e das repetições. Água e ração foram fornecidas $a d$ libidum durante todo o período experimental, sendo os bebedouros do tipo pressão e comedouros tubulares, com capacidade para $15 \mathrm{~kg}$. O aquecimento foi fornecido por meio de lâmpadas de 60 Watts, ligadas durante os primeiros dias do experimento. Realizou-se o manejo de temperatura interna dos boxes, de acordo com a temperatura ambiente e com o comportamento dos pintos. O programa de luz adotado foi o contínuo (natural + artificial), durante todo o período experimental.

Os dados de desempenho foram obtidos semanalmente e analisados nos períodos acumulados de um a 28 e de 29 a 90 dias de idade. Para obtenção do peso corporal, as aves de cada boxe foram pesadas juntas no alojamento e semanalmente até 90 dias de idade. O ganho de peso foi obtido pela diferença entre peso final e peso inicial das aves. O consumo de ração foi calculado pela diferença entre o total de ração consumida e as sobras de ração no final de cada período. A conversão alimentar foi o resultado da razão entre o total de ração consumida e o ganho de peso no período.

Tabela 1. Composição das dietas experimentais para frangos de corte tipo caipira na fase inicial (1-28 dias)

\begin{tabular}{|c|c|c|c|}
\hline \multirow{2}{*}{ Ingrediente (\%) } & \multicolumn{3}{|c|}{ Níveis de energia metabolizável (kcal/kg) } \\
\hline & 3.000 & 3.100 & 3.200 \\
\hline Milho moído & 58,83 & 56,54 & 54,25 \\
\hline Farelo de soja & 32,60 & 32,90 & 33,33 \\
\hline Óleo vegetal & 1,80 & 3,70 & 5,60 \\
\hline Farinha carne & 5,50 & 5,60 & 5,60 \\
\hline Calcário calcítico & 0,37 & 0,36 & 0,35 \\
\hline Sal comum & 0,30 & 0,30 & 0,30 \\
\hline Premix mineral e vitamínico ${ }^{(*)}$ & 0,60 & 0,60 & 0,60 \\
\hline Total & 100 & 100 & 100 \\
\hline \multicolumn{4}{|l|}{ Composição nutricional calculada } \\
\hline Energia metabolizável (kcal/kg) & 3.000 & 3.100 & 3.200 \\
\hline Proteína bruta $\%$ & 22,00 & 22,00 & 22,00 \\
\hline Extrato etéreo \% & 4,89 & 6,70 & 8,51 \\
\hline Fibra bruta $\%$ & 3,54 & 3,52 & 3,49 \\
\hline Matéria mineral \% & 5,31 & 5,31 & 5,31 \\
\hline \multirow{2}{*}{$\begin{array}{l}\text { Lisina \% } \\
\text { Cálcio \% }\end{array}$} & 1,21 & 1,22 & 1,23 \\
\hline & 1,00 & 1,00 & 1,00 \\
\hline Fósforo total \% & 0,68 & 0,68 & 0,68 \\
\hline Fósforo disponível \% & 0,48 & 0,48 & 0,48 \\
\hline Sódio \% & 0,17 & 0,17 & 0,17 \\
\hline
\end{tabular}

*Composição por kg do produto: vit. A - 1.835.000 UI; vit. D3 - 335.000 UI; vit. E - 2.835mg; vit. B1 - 335mg; vit. B2 - 1.000mg; vit. B6 - 335mg; vit. K3 - 417mg; vit. B12 - $2.500 \mu \mathrm{g}$; biotina - 17mg; ácido fólico - 135mg; niacina $-6.670 \mathrm{mg}$; selênio - 35mg; antioxidante $-2.000 \mathrm{mg}$; pantontenato de cálcio $-1.870 \mathrm{mg}$; cobre $-1.000 \mathrm{mg}$; cobalto 35mg; Iodo - 170mg; ferro $8.335 \mathrm{mg}$; manganês - 10.835mg; zinco - 8.335mg; cloreto de colina $50 \%$ 135.000; metionina - 267.000mg; coccdiostático - 13.335mg;

T1: 3.000kcal de EM/kg; T2: 3.100kcal de EM/kg; T3: 3.200kcal de EM/kg 
Tabela 2. Composição das dietas experimentais para frangos caipiras na fase final (29-90 dias)

\begin{tabular}{|c|c|c|c|}
\hline \multirow{2}{*}{ Ingrediente (\%) } & \multicolumn{3}{|c|}{ Níveis de energia metabolizável (kcal/kg) } \\
\hline & 3.100 & 3.200 & 3.300 \\
\hline Milho moído & 63,24 & 60,95 & 58,66 \\
\hline Farelo de soja & 27,20 & 27,60 & 27,90 \\
\hline Óleo vegetal & 2,60 & 4,50 & 6,40 \\
\hline Farinha carne & 5,70 & 5,70 & 5,80 \\
\hline Calcário calcítico & 0,36 & 0,35 & 0,34 \\
\hline Sal comum & 0,30 & 0,30 & 0,30 \\
\hline Premix mineral e vitamínico ${ }^{(*)}$ & 0,60 & 0,60 & 0,60 \\
\hline Total & 100,00 & 100,00 & 100,00 \\
\hline \multicolumn{4}{|l|}{ Composição nutricional calculada } \\
\hline Energia metabolizável (kcal/kg) & 3.100 & 3.200 & 3.300 \\
\hline Proteína bruta \% & 20,00 & 20,00 & 20,00 \\
\hline Extrato etéreo \% & 5,83 & 7,64 & 9,45 \\
\hline Fibra bruta $\%$ & 3,29 & 3,27 & 3,24 \\
\hline Matéria mineral \% & 5,08 & 5,08 & 5,08 \\
\hline Lisina $\%$ & 1,07 & 1,08 & 1,08 \\
\hline Cálcio \% & 1,00 & 1,00 & 1,00 \\
\hline Fósforo total \% & 0,67 & 0,66 & 0,66 \\
\hline Fósforo disponível \% & 0,48 & 0,48 & 0,48 \\
\hline Sódio \% & 0,17 & 0,17 & 0,17 \\
\hline \multicolumn{4}{|c|}{ 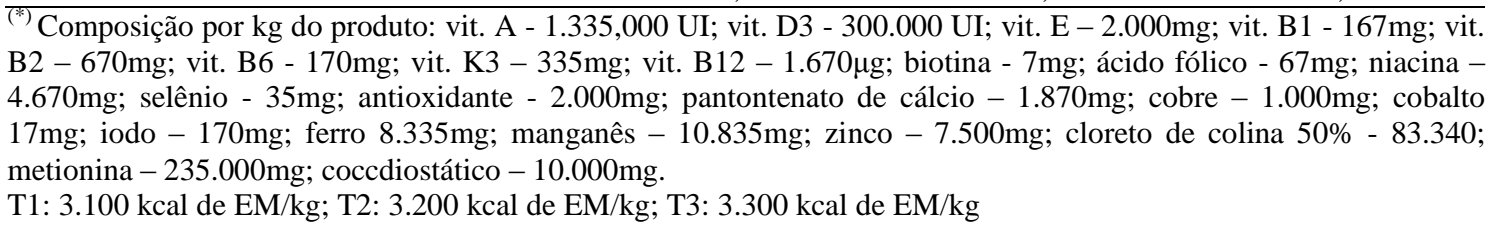 } \\
\hline
\end{tabular}

Duas aves por repetição de cada tratamento foram abatidas aos 77,84 e 90 dias de idade, após passarem por um jejum de 12 horas. Seguindo o processamento convencional de abate, realizou-se a coleta dos dados da pesquisa. Os cortes de coxas, sobrecoxas, peito, asas, dorso, pés, cabeça e pescoço foram pesados em balança com precisão de $5 \mathrm{~g}$, e seus rendimentos calculados em relação ao peso da ave ao abate. As variáveis estudadas para desempenho zootécnico foram peso médio, ganho de peso diário, consumo de ração e conversão alimentar; para características de carcaça foram rendimento de carcaça e cortes nobres (peito, coxa e sobrecoxa), peso relativo das asas, dorso, cabeça+pescoço e pés.

A análise estatística foi realizada utilizando-se o procedimento ANOVA, com o programa Sistema para Análises Estatísticas e Genética - SAEG (Sistema..., 2007). Para avaliar as diferenças entre médias das variáveis estudadas, usou-se teste de Tukey a $5 \%$ de probabilidade.

\section{RESULTADOS E DISCUSSÃO}

Os resultados de desempenho zootécnico são mostrados na Tab. 3. Verificou-se efeito significativo $(\mathrm{P}<0,05)$ dos níveis de energia metabolizável da ração na fase inicial sobre o peso médio, o ganho de peso e a conversão alimentar das aves do T3, no entanto não há diferença significativa $(\mathrm{P}>0.05)$ em relação às aves do $\mathrm{T} 2$.

Estes achados são similares aos encontrados por Oliveira Neto et al. (2000), os quais reportaram melhores índices de ganho de peso e conversão alimentar em aves da linhagem Hubbard, que consumiram a dieta com 3.232 e $3.224 \mathrm{kcal}$ de $\mathrm{EM} / \mathrm{kg}$, aos 22 dias de idade. Do mesmo modo, Hellmeister Filho et al. (2003), alimentando frangos caipiras das linhagens Pescoço Pelado de Piracicaba, Label Rouge e Paraíso Pedrês com rações contendo níveis de energia de 3.000 e $3.150 \mathrm{kcal}$ de EM/kg, também verificaram melhor desempenho de ganho de peso, consumo de ração e conversão alimentar. 
Tabela 3. Desempenho zootécnico de frangos da linhagem Caipira Francês Exótico alimentados com rações contendo diferentes níveis de energia metabolizável (EM), durante a fase inicial (1 a 28 dias) e final (29 a 90 dias)

\begin{tabular}{lcccc} 
Tratamento & $\begin{array}{c}\text { Peso médio } \\
(\mathrm{g})\end{array}$ & $\begin{array}{c}\text { Consumo de } \\
\text { ração (g/ave) }\end{array}$ & $\begin{array}{c}\text { Ganho de peso } \\
\text { (g/ave/dia) }\end{array}$ & $\begin{array}{c}\text { Conversão } \\
\text { alimentar }\end{array}$ \\
\hline T1 - 3.000kcal de EM/kg & $615,75 \mathrm{~b}$ & $1.102,00 \mathrm{a}$ & $22,80 \mathrm{~b}$ & $1,85 \mathrm{~b}$ \\
T2 - 3.100kcal de EM/kg & $655,75 \mathrm{ab}$ & $1.082,75 \mathrm{a}$ & $24,28 \mathrm{ab}$ & $1,77 \mathrm{ab}$ \\
T3 - 3.200kcal de EM/kg & $713,50 \mathrm{a}$ & $1.074,00 \mathrm{a}$ & $26,42 \mathrm{a}$ & $1,59 \mathrm{a}$ \\
CV $(\%)$ & 6,51 & 8,30 & 8,34 & 5,44 \\
\hline T1 - 3.100kcal de EM/kg & $3.493,12 \mathrm{a}$ & $6.228,25 \mathrm{a}$ & $47,17 \mathrm{a}$ & $2,16 \mathrm{a}$ \\
T2 - 3.200kcal de EM/kg & $3.455,04 \mathrm{a}$ & $5.944,75 \mathrm{a}$ & $45,89 \mathrm{a}$ & $2,12 \mathrm{a}$ \\
T3 - 3.300kcal de EM/kg & $3.658,58 \mathrm{a}$ & $6.433,50 \mathrm{a}$ & $48,28 \mathrm{a}$ & $2,18 \mathrm{a}$ \\
CV $(\%)$ & 5,38 & 8,48 & 6,54 & 6,36 \\
\hline
\end{tabular}

Médias seguidas de letras distintas na coluna diferem entre si pelo teste de Tukey $(\mathrm{P}<0,05)$.

Tratamento 1: 3.000 e 3.100kcal de EM/kg; Tratamento 2: 3.100 e 3.200kcal de EM/kg; Tratamento 3: 3.200 e $3.300 \mathrm{kcal}$ de $\mathrm{EM} / \mathrm{kg}$.

CV: coeficiente de variação.

Resultados diferentes foram reportados por Mendonça et al. (2004), que constataram uma queda linear do consumo de ração e na conversão alimentar à medida que os níveis de energia metabolizável aumentaram em dietas de frangos de corte da linhagem Isa Label. Estas aves receberam ração contendo níveis de energia que variavam de 2.600 a $3.200 \mathrm{kcal}$ de $\mathrm{EM} / \mathrm{kg}$ na fase inicial, 2.700 a $3.300 \mathrm{kcal}$ de EM/kg na fase de crescimento e 2.800 a $3.400 \mathrm{kcal}$ de EM/kg na fase final. Da mesma forma, Araújo et al. (2005) e Pacheco et al. (2004) também observaram melhora no ganho de peso e na conversão alimentar em frangos das linhagens Isa Label e Label Rouge, à medida que os níveis de energia metabolizável aumentaram.

Xavier et al. (2008) não observaram efeito significativo sobre o desempenho de frangos de corte da linhagem Cobb, submetidos a diferentes níveis de energia metabolizável $(2.850,2.950$, $3.000,3.045$ e $3.150 \mathrm{kcal}$ de EM/kg). Resultados semelhantes foram verificados por Duarte et al. (2007), que não encontraram influência dos níveis de energia sobre o ganho de peso, o consumo de ração e a conversão alimentar de frangos alimentados com 3.200 e $3.600 \mathrm{kcal}$ de $\mathrm{EM} / \mathrm{kg}$.

Não foi encontrado efeito significativo dos níveis de energia sobre o desempenho zootécnico das aves durante a fase final (29-90 dias). Resultados semelhantes foram verificados por Ávila et al. (2005), que não constataram influência significativa sobre o peso médio de frangos da linhagem Embrapa 41, submetidos a quatro níveis de energia metabolizável (2.600, 2.800, 3.000 e $3.200 \mathrm{kcal}$ de EM/kg). Da mesma forma, Sakomura et al. (2004) não observaram diferença significativa no consumo de ração, quando estudaram os efeitos de três níveis de energia da dieta $(3.050,3.200$ e $3.350 \mathrm{kcal}$ de $\mathrm{EM} / \mathrm{kg}$ de ração) em frangos de corte na fase de crescimento (22 a 43 dias de idade).

Os resultados quanto ao rendimento de carcaça e aos cortes nobres encontram-se na Tab. 4. Foi observado efeito significativo $(\mathrm{P}<0,05)$ dos níveis de energia metabolizável sobre o rendimento de carcaça e do peito das aves que consumiram dietas do $\mathrm{T} 2$, pois apresentaram melhores resultados, e não diferiram do $\mathrm{T} 1$.

Para rendimento de carcaça, constatou-se que as aves que consumiram dietas a 3.000 e $3.100 \mathrm{kcal}$ de EM/kg obtiveram melhores resultados quando abatidas aos 90 dias de idade. Ao consumirem a mesma dieta, as aves apresentaram melhores resultados de rendimento de peito quando abatidas aos 90 dias de idade, não diferindo, no entanto, dos resultados obtidos aos 77 dias. 
O peso relativo da sobrecoxa foi maior $(\mathrm{P}<0,05)$ nas aves que consumiram dieta com energia de 3.200 e $3.300 \mathrm{kcal}$ de $\mathrm{EM} / \mathrm{kg}$, abatidas aos 90 dias, diferindo estatisticamente das aves abatidas aos 77 e 84 dias de idade.

Estes resultados assemelham-se aos de Rosa et al. (2000) e Mendes et al. (2001), que também encontraram efeito dos níveis de energia da dieta sobre o rendimento de carcaça em frangos de corte. Todavia, divergem dos relatos de
Takahashi et al. (2006), que não observaram diferenças para rendimento de cortes comerciais em linhagens de frangos Pescoço Pelado e Paraíso Pedrês, aos 84 dias de idade, alimentados com dietas contendo 2.800 e $2.900 \mathrm{kcal}$ de $\mathrm{EM} / \mathrm{kg}$. Sakomura et al. (2004), utilizando diferentes níveis de energia metabolizável $(3.050,3.200$ e $3.350 \mathrm{kcal}$ de EM/kg) na dieta de frangos de corte na fase de crescimento, também não constataram efeito significativo sobre $o$ rendimento de carcaça.

Tabela 4. Rendimento de carcaça, peito, coxas e sobrecoxas de frangos da linhagem Caipira Francês Exótico alimentados com rações contendo diferentes níveis de energia metabolizável (EM), em três idades de abate

\begin{tabular}{|c|c|c|c|c|}
\hline \multirow[t]{2}{*}{ Tratamento } & \multicolumn{4}{|c|}{ Carcaça } \\
\hline & 77 dias & 84 dias & 90 dias & $\mathrm{CV}(\%)$ \\
\hline T1 - 3.000 e $3.100 \mathrm{kcal}$ de EM/kg & $80,72 \mathrm{abB}$ & $82,41 \mathrm{aAB}$ & $84,10 \mathrm{aA}$ & 3,52 \\
\hline $\mathrm{T} 2-3.100$ e $3.200 \mathrm{kcal}$ de $\mathrm{EM} / \mathrm{kg}$ & $82,20 \mathrm{aA}$ & $82,35 \mathrm{aA}$ & $79,87 \mathrm{aA}$ & 7,20 \\
\hline $\mathrm{T} 3-3.200$ e $3.300 \mathrm{kcal}$ de $\mathrm{EM} / \mathrm{kg}$ & $76,42 \mathrm{bA}$ & $81,36 \mathrm{aA}$ & $80,37 \mathrm{aA}$ & 6,78 \\
\hline \multirow[t]{2}{*}{$\mathrm{CV}(\%)$} & 5,64 & 9,76 & 8,74 & \\
\hline & \multicolumn{4}{|c|}{ Peito } \\
\hline $\mathrm{T} 1-3.000$ e $3.100 \mathrm{kcal} \mathrm{de} \mathrm{EM} / \mathrm{kg}$ & $23,20 \mathrm{abAB}$ & $21,60 \mathrm{aB}$ & $25,06 \mathrm{aA}$ & 9,10 \\
\hline $\mathrm{T} 2-3.100$ e $3.200 \mathrm{kcal}$ de EM $/ \mathrm{kg}$ & $24,26 \mathrm{aA}$ & $20,57 \mathrm{aA}$ & $23,27 \mathrm{aA}$ & 12,70 \\
\hline $\mathrm{T} 3-3.200$ e $3.300 \mathrm{kcal}$ de EM/kg & $21,84 \mathrm{bA}$ & $22,35 \mathrm{aA}$ & $23,16 \mathrm{aA}$ & 5,82 \\
\hline \multirow[t]{2}{*}{$\mathrm{CV}(\%)$} & 5,69 & 12,82 & 7,07 & \\
\hline & \multicolumn{4}{|c|}{ Coxas } \\
\hline $\mathrm{T} 1-3.000$ e $3.100 \mathrm{kcal}$ de EM/kg & $13,65 \mathrm{aA}$ & $14,13 \mathrm{aA}$ & $13,76 \mathrm{aA}$ & 9,64 \\
\hline $\mathrm{T} 2-3.100$ e $3.200 \mathrm{kcal}$ de EM/kg & $13,59 \mathrm{aA}$ & $13,73 \mathrm{aA}$ & $13,97 \mathrm{aA}$ & 8,25 \\
\hline $\mathrm{T} 3-3.200$ e $3.300 \mathrm{kcal}$ de $\mathrm{EM} / \mathrm{kg}$ & $13,73 \mathrm{aA}$ & $14,04 \mathrm{aA}$ & $13,85 \mathrm{aA}$ & 6,49 \\
\hline \multirow[t]{2}{*}{$\mathrm{CV}(\%)$} & 6,48 & 8,76 & 8,74 & \\
\hline & \multicolumn{4}{|c|}{ Sobrecoxas } \\
\hline T1 - 3.000 e $3.100 \mathrm{kcal}$ de EM/kg & $12,82 \mathrm{aA}$ & $13,30 \mathrm{aA}$ & $13,56 \mathrm{aA}$ & 5,80 \\
\hline $\mathrm{T} 2-3.100$ e $3.200 \mathrm{kcal}$ de EM/kg & $13,51 \mathrm{aA}$ & $12,88 \mathrm{aA}$ & $13,30 \mathrm{aA}$ & 11,27 \\
\hline $\mathrm{T} 3-3.200$ e $3.300 \mathrm{kcal}$ de $\mathrm{EM} / \mathrm{kg}$ & $12,60 \mathrm{aB}$ & $12,77 \mathrm{aB}$ & $13,62 \mathrm{aA}$ & 6,86 \\
\hline $\mathrm{CV}(\%)$ & 6,96 & 10,85 & 7,98 & \\
\hline
\end{tabular}

Médias seguidas por letras distintas minúsculas na coluna e maiúscula na linha diferem entre si pelo teste de Tukey $(\mathrm{P}<0,05)$

Tratamento 1: 3.000 e 3.100kcal de EM/kg; Tratamento 2: 3.100 e 3.200kcal de EM/kg; Tratamento 3: 3.200 e $3.300 \mathrm{kcal}$ de $\mathrm{EM} / \mathrm{kg}$.

CV: Coeficiente de variação.

Estes contrastes se justificam pelas diferenças entre linhagens avaliadas, níveis de energia utilizados e balanceamento diferenciado das dietas experimentais. Segundo Moreira et al. (2003), as variações no rendimento de carcaça e das parte em linhagens comerciais de frangos de corte observadas em algumas pesquisas também podem ser atribuídas às diferenças na taxa de crescimento e na idade de abate.

Os resultados de peso relativo das asas, dorso, pés e cabeça+pescoço estão apresentados na Tab. 5. Não foi verificada influência significativa ( $\mathrm{P}>0,05)$ dos níveis de energia metabolizável da ração sobre as variáveis estudadas. 
Tabela 5. Peso relativo das asas, dorso, pés, cabeça e pescoço de frangos da linhagem Caipira Francês Exótico alimentados com rações contendo diferentes níveis de energia metabolizável (EM), em três idades de abate

\begin{tabular}{|c|c|c|c|c|}
\hline \multirow[t]{2}{*}{ Tratamento } & \multicolumn{4}{|c|}{ Asas } \\
\hline & 77 dias & 84 dias & 90 dias & $\mathrm{CV}(\%)$ \\
\hline $\mathrm{T} 1-3.000$ e $3.100 \mathrm{kcal}$ de EM/kg & $10,56 \mathrm{~A}$ & $10,65 \mathrm{~A}$ & $10,15 \mathrm{~A}$ & 6,28 \\
\hline $\mathrm{T} 2-3.100$ e $3.200 \mathrm{kcal}$ de EM/kg & $10,63 \mathrm{~A}$ & $10,31 \mathrm{~A}$ & $10,31 \mathrm{~A}$ & 7,99 \\
\hline $\mathrm{T} 3-3.200$ e $3.300 \mathrm{kcal}$ de EM/kg & $10,52 \mathrm{~A}$ & $10,57 \mathrm{~A}$ & $10,59 \mathrm{~A}$ & 3,52 \\
\hline \multirow[t]{2}{*}{$\mathrm{CV}(\%)$} & 6,12 & 6,57 & 5,10 & \\
\hline & \multicolumn{4}{|c|}{ Dorso } \\
\hline T1 - 3.000 e $3.100 \mathrm{kcal}$ de EM/kg & $18,60 \mathrm{~B}$ & $23,56 \mathrm{~B}$ & $18,58 \mathrm{~B}$ & 17,61 \\
\hline $\mathrm{T} 2-3.100$ e $3.200 \mathrm{kcal}$ de $\mathrm{EM} / \mathrm{kg}$ & $21,06 \mathrm{~A}$ & $21,53 \mathrm{~A}$ & $19,48 \mathrm{~A}$ & 8,69 \\
\hline $\mathrm{T} 3-3.200$ e $3.300 \mathrm{kcal}$ de EM/kg & $21,69 \mathrm{~A}$ & $22,24 \mathrm{~A}$ & $19,68 \mathrm{~A}$ & 5,96 \\
\hline \multirow[t]{2}{*}{$\mathrm{CV}(\%)$} & 14,31 & 9,51 & 6,39 & \\
\hline & \multicolumn{4}{|c|}{ Pés } \\
\hline $\mathrm{T} 1-3.000$ e $3.100 \mathrm{kcal}$ de EM/kg & $4,87 \mathrm{~B}$ & $5,19 \mathrm{AB}$ & $4,21 \mathrm{~A}$ & 13,04 \\
\hline $\mathrm{T} 2-3.100$ e $3.200 \mathrm{kcal}$ de EM/kg & $4,72 \mathrm{~A}$ & $4,25 \mathrm{~A}$ & $4,24 \mathrm{~A}$ & 16,57 \\
\hline $\mathrm{T} 3-3.200$ e $3.300 \mathrm{kcal}$ de EM/kg & $4,85 \mathrm{~A}$ & $4,70 \mathrm{~A}$ & $4,32 \mathrm{~A}$ & 13,29 \\
\hline \multirow[t]{2}{*}{$\mathrm{CV}(\%)$} & 11,60 & 25,0 & 11,63 & \\
\hline & \multicolumn{4}{|c|}{ Cabeça + Pescoço } \\
\hline $\mathrm{T} 1-3.000$ e $3.100 \mathrm{kcal}$ de EM/kg & $11,95 \mathrm{~A}$ & $10,62 \mathrm{~A}$ & $11,33 \mathrm{~A}$ & 24,94 \\
\hline $\mathrm{T} 2-3.100$ e $3.200 \mathrm{kcal}$ de $\mathrm{EM} / \mathrm{kg}$ & $9,55 \mathrm{~A}$ & $10,31 \mathrm{~A}$ & $11,06 \mathrm{~A}$ & 11,85 \\
\hline $\mathrm{T} 3-3.200$ e $3.300 \mathrm{kcal}$ de $\mathrm{EM} / \mathrm{kg}$ & $10,76 \mathrm{~A}$ & $10,30 \mathrm{~A}$ & $10,99 \mathrm{~A}$ & 11,52 \\
\hline $\mathrm{CV}(\%)$ & 10,08 & 12,89 & 8,46 & \\
\hline
\end{tabular}

Médias seguidas por letras distintas minúsculas na coluna e maiúscula na linha diferem entre si pelo teste de Tukey $(\mathrm{P}<0,05)$.

Tratamento 1: 3.000 e 3.100kcal de EM/kg; Tratamento 2: 3.100 e 3.200kcal de EM/kg; Tratamento 3: 3.200 e $3.300 \mathrm{kcal}$ de $\mathrm{EM} / \mathrm{kg}$.

CV: Coeficiente de variação.

ns: não significativo $(\mathrm{P}>0,05)$ para idade de abate.

Este resultado assemelha-se ao encontrado por Mendes et al. (2004), que não constataram efeito dos níveis de energia sobre o rendimento de cortes em frangos submetidos a dietas contendo seis níveis de energia $(2.900,2.960,3.020$, $3.080,3.140$ e $3.200 \mathrm{kcal}$ de EM/kg). Ao analisar as diferentes idades de abate, constatou-se que as aves abatidas aos 84 dias apresentaram peso relativo de dorso e de pés significativamente superior $(\mathrm{P}<0,05)$ quando comparadas as demais idades.

\section{CONCLUSÕES}

Os frangos da linhagem Caipira Francês Exótico apresentaram melhores desempenho zootécnico e rendimento de carcaça quando alimentados com dietas contendo 3.100 a $3.200 \mathrm{kcal}$ de $\mathrm{EM} / \mathrm{kg}$ na ração e abatidos aos 77 dias de idade.

\section{REFERÊNCIAS}

ARAÚJO, L.F.; JUNQUEIRA, O.M.; ARAÚJO, C.S.S. et al. Energy and lysine for broilers from 44 to 55 days of age. Rev. Bras. Cienc. Avic., v.7, p.237$241,2005$.

ÁVILA, V.S.; COLDEBELLA, A.; FIGUEIREDO, E.A.P. et al. Frangos de corte tipo caipira ou colonial, "Isa Label", criados com diferentes níveis de energia metabolizável em dois sistemas de criação. Comunicado Técnico 394. Embrapa suínos e aves. Concórdia. 2005.

BASTIANELLI, D.A. Produção de frangos diferenciados na França: mercado, aspectos organizacionais e regulamentares. In: CONFERÊNCIA APINCO DE CIÊNCIA E TECNOLOGIA AVÍCOLAS, 2001, Campinas, SP. Anais... Campinas: FACTA, 2001. p.235-254.

DUARTE, K.F.; JUNQUEIRA, O.M.; FILARDI, R.S. et al. Efeito dos níveis de energia e programas de alimentação sobre a qualidade de carcaça e desempenho de frangos de corte abatidos tardiamente. Acta. Sci. Anim. Sci., v.29, p.39-47, 2007. 
FIGUEIREDO, E.A.P.; PAIVA, D.P.; ROSA, P.S. et al. Diferentes denominações e classificação brasileira de produção alternativa de frangos. In: CONFERÊNCIA APINCO 2001 DE CIÊNCIA E TECNOLOGIA AVÍCOLAS, 2001, Campinas, SP. Anais... Campinas: FACTA, 2001. p.209- 222.

FREITAS, E.R.; SAKOMURA, N.K.; EZEQUIEL, J.M.B. et al. Energia metabolizável de alimentos na formulação de ração para frangos de corte. Pesq. Agropec. Bras., v.41, p.107-115, 2006.

HELLMEISTER FILHO, P.; MENTEN, M.J.; SILVA, A.M. et al. Efeito de genótipo e do sistema de criação sobre o desempenho de frangos tipo caipira. Rev. Bras. Zootec., v.32, p.1883-1889, 2003.

LESSON, S.; SUMMERS, J.D. Nutrition of the chicken. 4. ed. Guelph: University Books, p. 413, 2001.

MENDES, A.A.; MOREIRA, J.; GARCIA, R.G. et al. Avaliação do rendimento e qualidade da carne de peito em frangos de corte criados com diferentes densidades e níveis de energia na dieta. Rev. Bras. Cienc. Avic., p.38, 2001.

MENDES, A.A.; MOREIRA, J.; OLIVEIRA, E.G. et al. Efeito da energia da dieta sobre o desempenho, rendimento de carcaça e gordura abdominal de frangos de corte. Rev. Bras. Zootec., v.33, p.2300-2307, 2004.

MENDONÇA, M.; SOKOMURA, N.; SANTOS, F. et al. Níveis de energia metabolizável e relações energia:proteína para aves de corte de crescimento lento criadas em sistema semiconfinado. Acta. Sci. Anim. Sci., v.29, p.23-30, 2007.

MOREIRA, J.; MENDES, A.A.; GARCIA, E.A. et al. Avaliação de desempenho, rendimento de carcaça e qualidade da carne do peito em frangos de linhagens de conformação versus convencionais. Rev. Bras. Zootec., v.32, p.1663-1673, 2003.

OLIVEIRA NETO, A.R.; OLIVEIRA, R.F.M.; DONZELE, J.L. et al. Efeito da temperatura ambiente sobre o desempenho e características de carcaça de frangos de corte alimentados com dieta controlada e dois níveis de energia metabolizável. Rev. Bras. Zootec., v.29, p.183-190, 2000.
PACHECO, O. Efeitos de diferentes níveis de energia e proteína sobre o desempenho de frangos de corte de linhagens coloniais. 2004. 40f. Dissertação (Mestrado em Ciências Veterinárias) - UFPR, Universidade Federal do Paraná. Curitiba.

ROSA, A.P.; BORIN Jr., H.; THIER J. et al. Desempenho e composição de carcaça de frangos submetidos a dietas com diferentes teores energéticos e níveis de gordura. In: REUNIÃO ANUAL DA SOCIEDADE BRASILEIRA DE ZOOTECNIA, 37., 2000, Viçosa, MG. Anais... Viçosa, MG: SBZ, 2000. p.228.

SAGRILO, E. (Ed.). Agricultura familiar. Teresina: Embrapa Meio-Norte, 2002. 74 p.

SAKOMURA, N.K.; LONGO, F.A.; ROBELLO, C. B. et al. Efeito do nível de energia metabolizável da dieta no desempenho e metabolismo energético de frango de corte. Rev. Bras. Zootec., v.33, p.1758-1767, 2004.

SISTEMA para Análises Estatísticas - SAEG, versão 9.1: Fundação Arthur Bernardes - UFV - Viçosa, 2007.

TAKAHASHI, S.E. Efeito do sistema de criação sobre o desempenho e qualidade da carne de frangos de corte tipo colonial e industrial. 2003. 64f. Dissertação (Mestrado em Zootecnia) - UNESP, Universidade Estadual Paulista, Botucatu.

TAKAHASHI, S.E.; MENDES, A.A.; SALDANHA, E.S.P.B. et al. Efeito do sistema de criação sobre o desempenho e rendimento de carcaça de frangos de corte tipo colonial. Arq. Bras. Med. Vet. Zootec., v.58, p.624-632, 2006.

VIANA, C.F.A.; SILVA, M.A.; PIRES, A.V. et al. Influência de Grupos Genéticos e de Níveis de Energia sobre Características de Carcaça de Frangos de Corte. Rev. Bras. Zootec., v.29, p.1076-1073, 2000.

XAVIER, S.A.G.; STRINGHINI, J.H.; BRITO, A.B. et al. Níveis de energia metabolizável em rações iniciais para frangos de corte. Rev. Bras. Zootec., v.37, p.109-115, 2008. 